\section{A CASE OF CHYLOTHORAX}

BY

MURIEL E. BELL, M.D.

ASSISTANT PATHOLOGIST, ROYAL INFIRMIARY, SUNDERLAND

(With Special Plate)

In view of the small number of recorded cases of chylous effusions into the thorax, the following may be of interest. The subject of chylous effusions has been fully treated by Wells $^{1}$ and by Powell and Hartley. ${ }^{2}$ It is therefore unnecessary to review cases previously reported in the literature.

\section{Clinical Case History}

The patient, a woman of 49 , complained of shortness of breath on exertion, abdominal discomfort, loss of weight, and gradually increasing weakness over a period of eight months. The chest was found to contain a turbid fluid which, on examination, bore the characteristics of a chylous effusion.

For the patient's comfort frequent withdrawal of fluid from both sides of the chest, and latterly from the abdomen, became necessary. During the fourteen weeks throughout which she was under observation in the ward 971 ounces of chylous fluid were removed, making a weekly average of sixty-nine ounces. This weekly figure falls considerably short of what is believed to be the amount of lymph entering the subclavian vein, even allowing that food intake and digestive processes were at a low level. It seemed justifiable to conclude, therefore, that the block in the thoracic duct was only partial-a surmise which was confirmed subsequently at postmortem examination.

\section{Hypothesis of Mechanism}

The cause of the chylothorax appeared in all probability to be a metastatic deposit in, or in juxtaposition to, the thoracic duct causing partial obstruction, the situation of this block being somewhere above the diaphragm. It was considered that the sequence of events was probably, in the first place, increased pressure in the lymphatics, distension of the lymphatic tributaries, which, being richly endowed with valves, would become nodular. Presumably the second event-namely, rupture-occurred as a result of friction of the pleural surfaces, combined with the negative pressure on the pleural surface of these swellings and an increased pressure inside the lymphatic capillaries. A small valvular opening being present the negative pressure accentuated with each respiration would cause a small amount of chyle to be sucked into the thorax; possibly the opening remained inactive during expiration.

There is no difficulty in explaining the bilateral effusion, and no need to postulate a cross-communication between the two pleural cavities. If the process had taken place on the one side the distress created by the presence of the fluid would deepen the movements on the other side of the chest, and the process would thus be repeated.

It was predicted that, with increase in the blockage of the duct, the positive pressure in the lymphatics of the abdominal cavity would rise to a sufficient height to cause rupture of the abdominal tributaries. It will be observed that chylous ascites occurred some weeks after the chylothorax. It would not have been surprising, from the situation of the growth as found at post-mortem examination, if it had occurred earlier by direct involvement of the lymphatics in front of the lumbar vertebrae. Chylous ascites is reported as occurring more frequently than chylothorax; the reason which suggests itself is that the obstruction occurs more frequently below the diaphragm. It would seem, at least on theoretical grounds, that if the block occurs above the diaphragm the negative pressure of the thorax tends to determine an earlier rupture of the thoracic lymphatics than of the abdominal channels. No chyluria supervened in this case ; the urine remained free from chyle and lymphocytes.

\section{Laboratory Findings}

The specific gravity of the fluid was 1018. When first withdrawn the fluid usually had a slight pink tint owing to the presence of a few red cells. On standing, however, the natural very pale yellow colour became apparent. The rapidity with which large amounts of fluid collected seemed to exclude the possibility of the effusion being pseudochylous. On the other hand, the volumes being much smaller than those one would expect from the total lymph flow from the thoracic duct into the subclavian vein, it was concluded that the block was only a partial one.

Turbidity and Cytology

There was a uniform turbidity, unaffected by filtration through paper, through a Berkefeld filter, or by centrifugalization. Only when the fluid had been treated by ether did the turbidity disappear; the ether extract was coloured, and on evaporation of the ether a fatty residue was left (see accompanying paper by Drummond, Bell, and Palmer, p. 1208). The percentage of fat, determined by ether extraction after drying with plaster-of-Paris, was variable. It became less as discomfort increased and appetite diminished. When the fluid had stood for a few days a cream rose to the top.

Lymphocytes were always present. The finely emulsified fat gave the fluid a granular appearance under the microscope, but there were no droplets to be seen and no cholesterol crystals.

The fluid did not coagulate spontaneously, except in the case of the first sample withdrawn from each pleural cavity. Subsequent specimens consistently failed to clot. It remained for a long time without undergoing decom. position. The analysis was as follows:

\begin{tabular}{|c|c|c|c|c|c|c|}
\hline Total solids & $\cdots$ & $\cdots$ & $\cdots$ & $\cdots$ & $\cdots$ & 6.025 \\
\hline Nitrogen & $\ldots$ & $\cdots$ & $\ldots$ & $\cdots$ & $\ldots$ & 0.98 \\
\hline Serum globulin & $\ldots$ & $\cdots$ & $\cdots$ & $\ldots$ & $\cdots$ & 2.07 \\
\hline Serum albumin & $\ldots$ & $\cdots$ & $\cdots$ & $\cdot$ & • & 2.41 \\
\hline Total protein & $\ldots$ & $\cdots$ & $\ldots$ & $\ldots$ & $\ldots$ & 4.48 \\
\hline Inorganic ash & ... & $\cdots$ & $\ldots$ & $\ldots$ & $\ldots$ & 0.79 \\
\hline Fat ... $\ldots$ & $\ldots$ & variable, & 0.61 , & 0.85 & 0.18 , & 0.23 \\
\hline Cholesterol... & ... & .. & $\ldots$ & $\ldots$ & $\ldots$ & 0.061 \\
\hline Sugar $\quad \ldots$ & ... & ... & $\ldots$ & 130 , & .106 & 0.132 \\
\hline
\end{tabular}

\section{Differential Diagnosis}

Certain conditions other than malignancy which might have been responsible for the chylous or pseudochylous effusion were excluded because no enlargement of the glands of the hilum was detected on $x$-ray examination, there was no evidence of leukaemic conditions, and inoculation of guinea-pigs was negative for tuberculosis.

Much has been written in discussions on the difference between chylous and pseudochylous fluids, though some of the distinctive features alleged to exist between the two varieties are non-proven. In the case recorded here there were two points which seemed to distinguish this as a true chylous leak-namely, the amount produced and the presence of lymphocytes.

\section{Post-mortem Findings}

The thoracic duct was dissected out in the neck and injected with $40 \mathrm{c} . \mathrm{cm}$. of lipiodol. By $x$-ray photograph the duct was seen to be of even calibre throughout its thoracic course (Fig. 1 on Plate). As the injection did not travel back into the smaller pulmonary tributaries the leak points could not be detected, and it was assumed that, as previously suspected, the openings were small and valvular. The duct appeared by comparison with that in other subjects to be dilated, except at the point of entry 
into the subclavian vein. Here a probe was passed with some difficulty; a nodule was felt at the junction, and on microscopical examination a collection of tumour cells surrounded and constricted the duct, and was projecting into the subclavian vein (Fig. 2 on Plate). A diagrammatic representation of the specimen is given below.

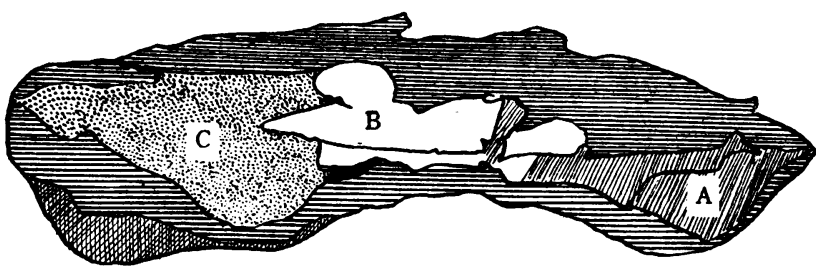

A, Thoracic duct. B, Growth. C, Subclavian vein.

Diagram of post-mortem specimen (see Fig. 2 on Plate).

The growth was found to be a retroperitoneal sarcoma of the small, round-celled type. It was growing densely in front of the lumbar vertebrae, invading the mesentery and the left kidney from below, and commencing to infiltrate the uterus and ovaries. There was no growth, either visible or microscopical, in the lungs or mediastinum.

A very small amount of fluid had collected in the abdominal cavity, though four days had elapsed since the abdomen had been tapped. This was to be contrasted with the condition found in the thorax, where, in spite of withdrawal of fluid on the day of death, about one and a half pints were present in each pleural cavity. This confirmed the belief that the main factor in determining the chylothorax had been the aspiration due to negative pressure in the thorax.

\section{Summary}

A case of bilateral chylothorax followed by chylous ascites is described. The symptoms corresponded with a condition arising from partial occlusion of the thoracic duct by a growth, and the diagnosis was confirmed at the post-mortem examination, when it was found that the junction of the thoracic duct with the subclavian vein was partially occluded by a metastatic deposit from a retroperitoneal sarcoma. The points of leakage in the chest and abdomen were believed to be small and valvular, but they were not definitely located at postmortem examination either by dissection or by injection of lipiodol.

Grateful appreciation is herewith expressed to Dr. Hamilton Ross for permission to study this case, which was under his care. Dr. H. A. Cookson, pathologist, is thanked for providing laboratory facilities, and for his helpful advice and criticism. Dr. Sharp kindly provided clinical notes and the supply of fluid from numerous tappings. Dr. Paige Arnold and his staff gave assistance by taking the $x$-ray and other photographs.

\section{REFERENCES}

${ }^{2}$ Wells, H. G.: Chemical Pathology, fifth edition, Philadelphia, 1925.

${ }^{2}$ Powell, R. D., and Hartley, P.: Diseases of the Lungs and Pleura sixth edition, London, 1921.

An international medical week will be held at Montreux, Switzerland, from September 9th to 14 th, to discuss subjects of practical interest to general medical practitioners. The discussions will be introduced by leading authorities from various countries. Sir Henry Dale will be one of the lecturers, the others including Professors L. Rajchman of the League of Nations, E. Bürgi of Berne, J. Holmgren of Stockholm, C. Nicolle of Tunis, Ximenez Diaz of Madrid, H. E. Sigerist of Baltimore, and M. Askanazy of Geneva. The subjects for discussion will be notified later. The fee for membership is 10 Swiss francs, and it will include payment for the report of the "Transactions." Further information may be obtained from the secretary of the International Week, Klosterberg 27, Basle.

\section{TUBERCULIN IN THE TREATMENT OF CUTANEOUS TUBERCULOSIS}

\author{
BY
}

H. S. BURNELL-JONES, D.P.H.OxoN

(With Special Plate)

Details are recorded in the Proceedings of the Royal Society of Medicine for January, 1934, and in the British Journal of Dermatology and Syphilis for February, 1934, of the treatment of three cases of cutaneous tuberculosis with tuberculin, the results of which encouraged continuation of the investigation. An account is presented here of three further cases similarly treated (out of a series referred from the skin department of the Royal Northern Hospital), for the purpose of encouraging further work and investigation.

Three tuberculins have been used-T.A.F., B.E., and T.R., obtained from Messrs. Bayer Ltd. In changing from one tuberculin to the other no particular rule has been followed, and no definite evidence has accumulated to indicate that one tuberculin is superior to another. Latterly I have been inclined to modify this statement in favour of T.A.F. and B.E. One fact that stands out pre-eminently-as pointed out by others-is that each case is a law unto itself with regard to intervals between injections, increase and decrease of dose, and the change of tuberculin, and that clinical insight and experience have to be depended upon.

\section{Technique Followed}

The site of injection is of importance ; patients showing local reactions on the arm will often show no local reaction if injected between the shoulders. The leg appears to be as sensitive as the arm. After experimenting with increases varying from 5 to $\mathbf{5 0}$ per cent., results have led to the opinion that 10 per cent. (or thereabouts) is the most satisfactory increase for dosage. The effectiveness of this small increase in skin cases, as compared with that feasible in pulmonary cases, may be due to a smaller quantity of lysin present in the tissue. In contradistinction to pulmonary tissue, the skin is not well flushed with blood, which results in a smaller supply of oxygen and pabulum, and perhaps accounts for relatively slower growth of the bacillus in this situation.

For the small doses a Koch's syringe is most convenient. The varying doses can be measured at the time of injection from the 1 in 10,1 in 100 , and 1 in 1,000 dilutions with the ordinary $0.1 \mathrm{c} . \mathrm{cm}$. pipette graduated in tenths. For doses of $0.2 \mathrm{c.cm}$. or over the ordinary $1 \mathrm{c} . \mathrm{cm}$. tuberculin syringe, graduated in tenths, is quicker. The dose of tuberculin is first drawn in, followed by the diluting fluid. The diluent is normal saline with 0.2 per cent. phenol, not 0.5 per cent. phenol. To the tongue 0.5 per cent. is distinctly appreciable, and in patients hypersensitive to phenol may account for the doubtful positive reaction sometimes seen after an intradermal test, which is followed by negative subcutaneous test injections when a positive temperature reaction might have been expected. No cleansing of the skin is done and no dressing is applied. Distilled water is used in the small metal sterilizer to prevent deposition of calcium salts in the syringes, needles, and pipettes. The glass pipettes are sterilized in a naked flame after rinsing with the distilled water in the sterilizer. The water should not be ejected back into the sterilizer. From time to time it is useful to clear the pipettes with pure nitric acid.

Routine intradermal and subcutaneous test doses are given in all cases. Valuable information is obtained by these preliminary injections as to the degree of allergy and the approximate dose with which treatment should bo 
MURIEL E. BELL : A CASE OF CHYLOTHORAX

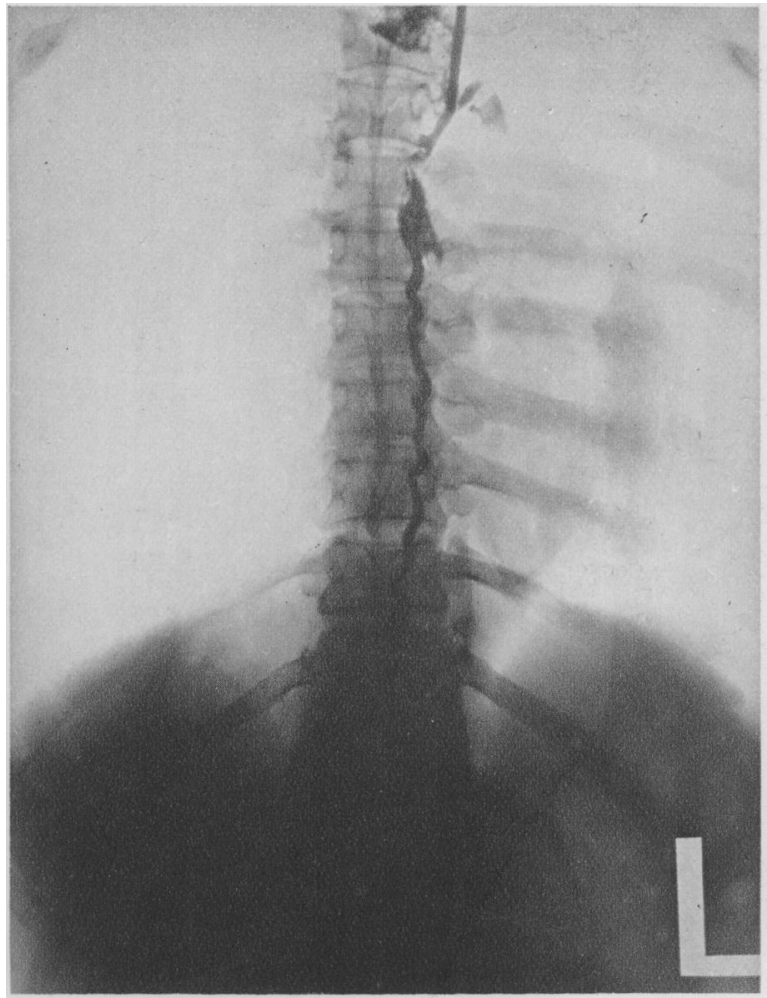

Fia. 1.- $X$-ray photograph of thoracic duct after injection of lipiodol. No interruption is seen in the main channel, but the injection did not travel back into the small vessels.

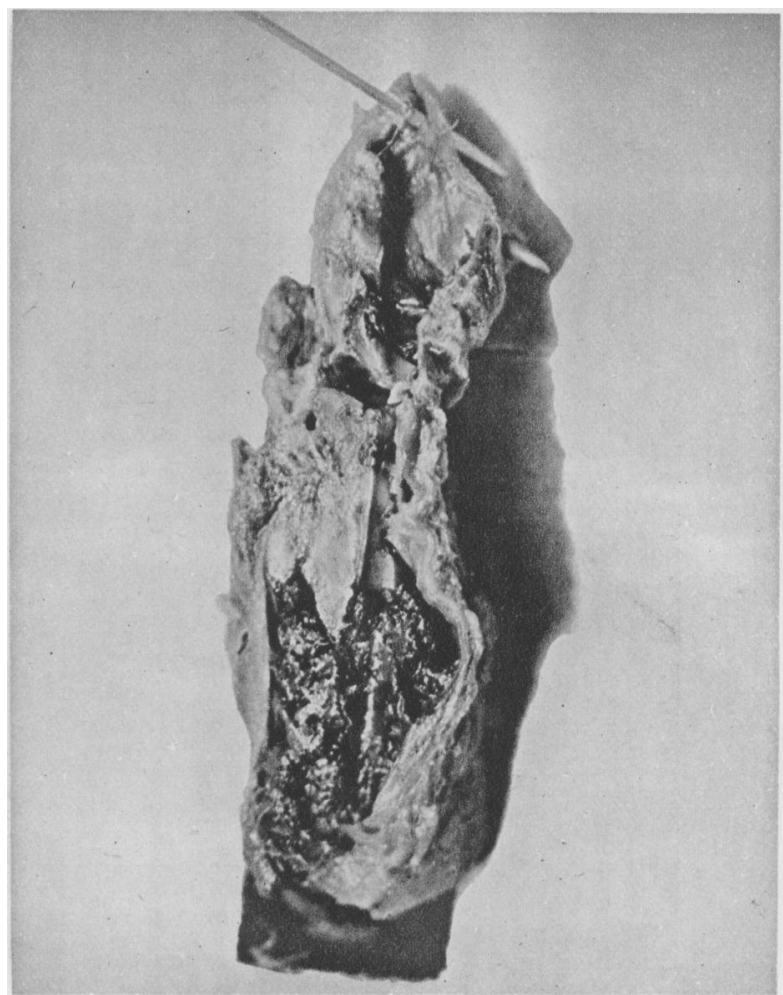

Fig. 2.-Photograph of the tumour growth surrounding and constricting the thoracic duct at the point of entry into the subclavian vein.

\section{J. FRFUD, D. LUWISCH, AND F. OESTREICHER: ULCERATIONS IN THE STOMACH AFTER ADRENALECTOMY}

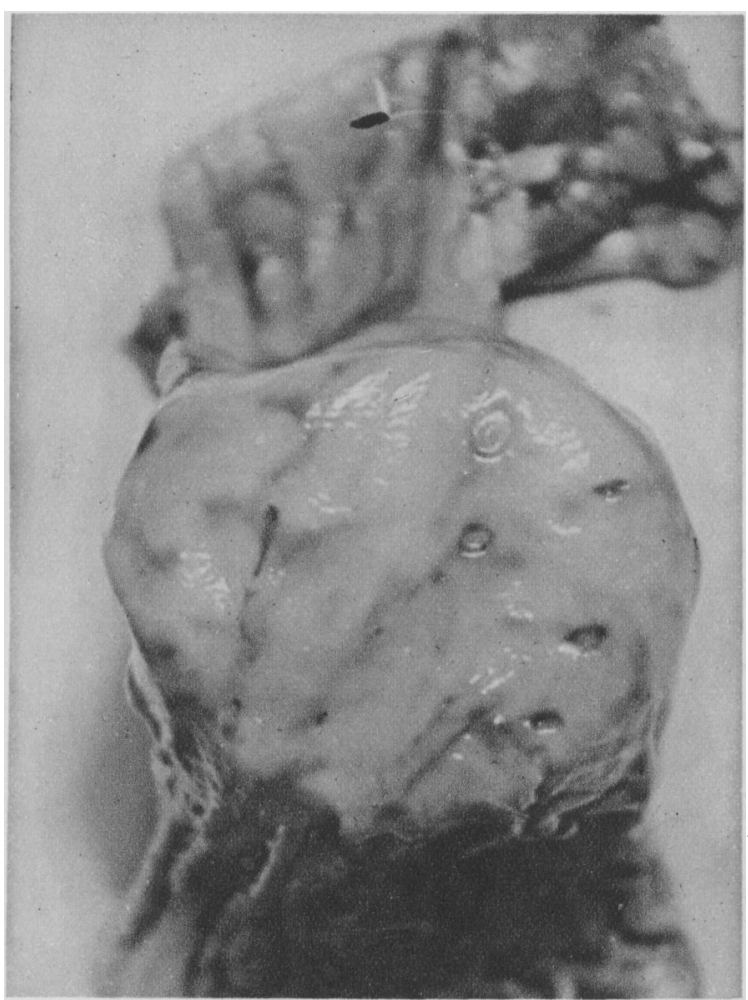

Fia. 1.-Pyloric end of the stomach with multiple -ulcerations.

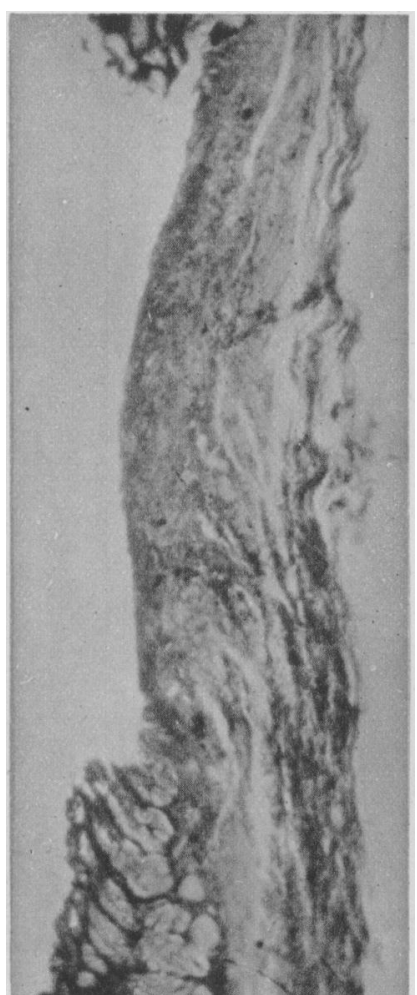
FIG. 2.- Microscoplcal picture of
the largest ulcer at low magnification.

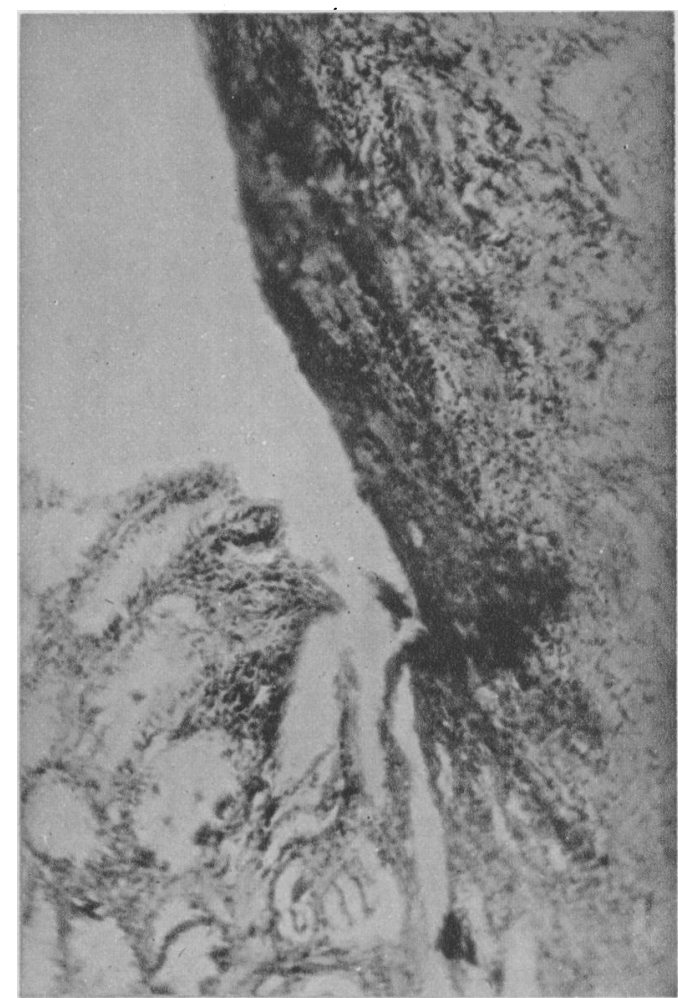

Fis. 3.-Margin of the ulcer at high-power magnifleation. 